\title{
Instantaneous Assessment of Hedonic Olfactory Perception Using Heartbeat Nonlinear Dynamics: A Preliminary Study
}

\author{
Alberto Greco ${ }^{1}$, Mimma Nardelli ${ }^{1}$, Antonio Lanata ${ }^{1}$, Maria Sole Morelli ${ }^{1}$, Fabio Di Francesco ${ }^{2}$, Enzo \\ Pasquale Scilingo ${ }^{1}$, Riccardo Barbieri ${ }^{2}$, and Gaetano Valenza ${ }^{1}$ \\ ${ }^{1}$ Bioengineering and Robotics Research Center "E. Piaggio" \& Dept. of Information Engineering, \\ University of Pisa, Pisa, Italy; ${ }^{2}$ Dept. of Chemistry and Industrial Chemistry, University of Pisa, Pisa, \\ Italy; ${ }^{3}$ Dept. of Electronics, Informatics and Bioengineering, Politecnico di Milano, Milano, Italy
}

\begin{abstract}
Emotional perception to hedonic olfactory stimuli is under direct control of the limbic system, whose dynamics is known to affect autonomic nervous system activity on cardiovascular control. Mainly due to methodological limitations, previous investigations failed to uncover specific trends in heartbeat dynamics between ultra shorttime (i.e., lasting $<10 s$ ), pleasant and unpleasant olfactory stimuli. To this extent, in this study we computed instantaneous estimates from heartbeat series gathered from 32 healthy subjects (age: 26士2; 16M) undergoing hedonic olfactory elicitation. Each subject exhibited a similar olfactory perception threshold, and scored five $5 \mathrm{~s}$ stimuli in terms of arousal and valence level using the selfassessment manikin test. We analyzed the heartbeat series using our recently proposed inhomogeneous point-process nonlinear framework, obtaining instantaneous estimates defined in the time (mean and standard deviation), frequency (power in the LF and HF bands, as well as LF/HF ratio), and nonlinear/complexity (bispectra, sample and approximate entropy, and Lyapunov exponent) domains. A feature set comprising average estimates within the $5 \mathrm{~s}$ windows was taken as an input of a K-Nearest Neighborhood classification algorithm, whose cross-validation relied on a leave-one-subject-out procedure. Results demonstrate that our framework allows to finely characterize affective olfactory elicitation with an average recognition accuracy of $71.88 \%$. Feature selection highlighted that the most discriminating power was contributed by instantaneous $L F$ power, instantaneous Lyapunov exponents, and instantaneous approximate entropy.
\end{abstract}

\section{Introduction}

Olfactory perception is a very complex phenomenon strongly linked to emotions [1]. Indeed, many fragrances are known to affect human emotional states, whose primary response is often described as enjoyable or annoying [1]. This has anatomical foundations in the connection between the olfactory neural path and brain areas such as amygdala and hippocampus, known to be involved in emotional processing [2]. As these limbic regions are also involved in the regulation of the endocrine system and the Autonomic Nervous System (ANS), the current literature consistently reports on changes in peripheral physiological dynamics during olfactory perception [3-8]. Particularly, a study on food fragrances showed that an early ANS response (after 1s) reflects the odors arousal, whereas delayed ANS responses (after 3-4s) reflect the odors valence [3]. Also, odor pleasantness correlated with heart rate variations [5,9], and flower smells induced a significant increase in the parasympathetic activity [4].

Nevertheless, also due to methodological limitations, changes in heartbeat dynamics throughout pleasant and/or unpleasant olfactory perception are not fully understood yet. Previous investigations, in fact, failed to report different (either linear or nonlinear/complex) heartbeat dynamics between ultra short-time (i.e., lasting less 10s), pleasant and unpleasant olfactory stimuli. In addition, classification models devised to automatically recognize hedonic differences between odors using heartbeat dynamics have not achieved sufficient accuracy [10].

To overcome these limitations, we here propose a novel framework using heartbeat dynamics estimated from inhomogeneous nonlinear point-process models [11-13], able to measure and classify olfactory perception. This methodological framework allows for the estimation of instantaneous ANS pattern associated with hedonic odorants, whose perception lasts for just a few seconds. Such estimates are exploited by feeding a classification algorithm to automatically discern the pleasantness associated with different olfactory stimuli.

\section{Materials and Methods}

\subsection{Point-Process Nonlinear Framework at a glance}

Our recently proposed point-process nonlinear framework for processing heartbeat dynamics aims at providing continuous estimates from the intrinsically discrete, un- 
evenly sampled heartbeat events detected from the electrocardiogram (ECG). Each heartbeat event (i.e., R-peak) is associated with a continuous Inverse-Gaussian probability density function (PDF) in time, from which it is possible to derive measures defined in the time (mean and standard deviation), frequency (power in the LF and HF bands, as well as LF/HF ratio), and nonlinear/complexity (bispectra, sample and approximate entropy, and Lyapunov exponent) domains, at any desired time resolution. Extensive methodological and mathematical details can be found in [11-13]. To summarize briefly, the PDF $f\left(t \mid H_{t}, \xi(t)\right)$, characterizing each heartbeat event, is parametrized in its time-varying parameter vector $\xi(t)=$ $\left[\xi_{0}^{a}(t), g_{0}(t),\left\{g_{1}(i, t)\right\}\right.$, and $\left.\left\{g_{2}(i, j, t)\right\}\right]$ with $\xi_{0}^{a}(t)>0$ as the shape parameter of the Inverse-Gaussian distribution, the history of the past heartbeat events $H_{t}^{a}=$ $\left(R R_{j}, R R_{j-1}, \ldots, R R_{j-p+1}\right)$, and by its first-order moment $\mu_{\mathrm{RR}}\left(t, H_{t}, \xi(t)\right)$ defined as a Nonlinear Autoregressive model with Laguerre expansions of the WienerVolterra terms:

$$
\begin{array}{r}
\mu_{\mathrm{RR}}\left(t, H_{t}, \xi(t)\right)=\mathrm{RR}_{\widetilde{\mathrm{N}}(\mathrm{t})}+\mathrm{g}_{0}(\mathrm{t})+\sum_{\mathrm{i}=0}^{\mathrm{p}} \mathrm{g}_{1}(\mathrm{i}, \mathrm{t}) \mathrm{l}_{\mathrm{i}}\left(\mathrm{t}^{-}\right)+ \\
\sum_{n=2}^{\infty} \sum_{i_{1}=1}^{M_{1}} \cdots \sum_{i_{n}=1}^{M_{n}} g_{n}\left(i_{1}, . ., i_{n}, t\right) \prod_{j=1}^{n} l_{i_{j}}\left(t^{-}\right)
\end{array}
$$

where $l_{i}\left(t^{-}\right)=\sum_{n=1}^{\widetilde{N}(t)} \phi_{i}(n)\left(\mathrm{RR}_{\widetilde{\mathrm{N}}(\mathrm{t})-\mathrm{n}}-\mathrm{RR}_{\widetilde{\mathrm{N}}(\mathrm{t})-\mathrm{n}-1}\right)$ is the output of the Laguerre filters just before time $t$, and $\phi_{i}(n)$ is the $i^{t h}$-order discrete time orthonormal Laguerre function. Considering the derivative R-R series improves the achievement of stationarity within the sliding time window $W$. We used a Newton-Raphson procedure to maximize the local log-likelihood defined in [11]. This formulation is also used to preprocess all the actual heartbeat data with a previously developed algorithm [14], performing a real-time R-R interval error (e.g., peak detection errors and ectopic beats) detection and correction. We determine the optimal model orders based on the Kolmogorov-Smirnov (KS) test and associated KS statistics [11]. Autocorrelation plots are also considered to test the independence of the model-transformed intervals [11]. As $\mu_{\mathrm{RR}}\left(t, H_{t}, \xi(t)\right)$ is defined in continuous time, it is possible to obtain an instantaneous mean estimate at a very fine timescale, which requires no interpolation between the arrival times of two beats. In this study, we have chosen $W=90 \mathrm{~s}$, Laguerre parameter $\alpha=0.2$, and $\mathrm{n}=3$ to account for nonlinearities associated to eq. 1 up to the third-order [11]. Cubic terms, in fact, allow for the estimation of the Lyapunov spectra, whereas quadratic terms account for the time, frequency, and higher-order spectral domains [11-13].

\subsubsection{Instantaneous estimates}

The time-domain characterization is based on the first and the second order moments of each inverse-Gaussian PDF associated with each heartbeat [11]. Given the time- varying parameter set $\xi(t)$, the instantaneous estimates of mean $\mu_{\mathrm{RR}}\left(t, H_{t}, \xi(t)\right)$ and instantaneous standard deviation $\sigma_{R R}\left(t, H_{t}, \xi(t)\right)$ can be derived [11].

The frequency domain characterization relies on the estimation of instantaneous linear power spectrum $Q\left(f, t, H_{t}, \xi(t)\right)[11]$. By integrating the $Q\left(f, t, H_{t}, \xi(t)\right)$ in the appropriate frequency bands, we can compute the instantaneous index within the low frequency $(\mathrm{LF}=0.05$ $0.15 \mathrm{~Hz})$, and high frequency $(\mathrm{HF}=0.15-0.5 \mathrm{~Hz})$ ranges.

The instantaneous presence of nonlinearity in heartbeat series can be revealed by calculating nonlinear sympatho-vagal interactions through instantaneous bispectrum $\left|\operatorname{Bis}\left(\mathrm{f}_{1}, \mathrm{f}_{2}, \mathrm{t}\right)\right|[11]$. By integrating in the appropriate frequency bands, it is possible to obtain bispectral markers of low-bispectral frequency interactions $\mathrm{LL}(\mathrm{t})$, lowhigh bispectral frequency interactions $\mathrm{LH}(\mathrm{t})$, and highbispectral frequency interactions $\mathrm{HH}(\mathrm{t})$. Further details on the instantaneous bispectrum derivation from pointprocess nonlinear models can be found in [11].

Instantaneous measures of heartbeat complexity can be obtained by considering cubic nonlinearities (with $n=3$ ) in the fully autoregressive eq. 1. Particularly, using a Fast Orthogonal Search algorithm, it is possible to estimate the complete Lyapunov Exponents (LE) spectrum at each moment in time [13] (in this study, we use the first and second exponents of the LE spectrum, i.e., $\lambda_{1}, \lambda_{2}$ ), as well as instantaneous entropy measures known as inhomogeneous point-process approximate and sample entropy, $A_{I}$ and $S_{I}$, respectively [12]. These measures have their foundation in the instantaneous phase space estimation, whose details can be found in $[12,13]$

\subsection{Subject Recruitment and Experimen- tal protocol}

Thirty-two volunteers, aged $26 \pm 2$ (16 males), gave their informed consent to be enrolled in the study. We designed an experiment where the subjects underwent to an affective elicitation by means of two odors, which were synthesized in the laboratory:

$\mathrm{O}_{1}$ : Benzaldehyde $\mathrm{C}_{6} \mathrm{H}_{5} \mathrm{CHO}$, (concentration 106.12 $\mathrm{g} / \mathrm{mol})$;

$\mathrm{O}_{2}$ : Isovaleric acid $(\mathrm{CH} 3)_{2} \mathrm{CHCH}_{2} \mathrm{COOH}$, (concentration $102.13 \mathrm{~g} / \mathrm{mol}$ ).

These odorants were selected due to their different hedonic tone (a pleasant bitter almond smell for benzaldehyde, an unpleasant sweat odor for isovaleric acid) and their safety [15]. The concentration of the solutions were chosen so that stimuli of similar intensity could be obtained. Participants were selected according to their olfactory threshold to N-butanol $\left(\mathrm{CH}_{3} \mathrm{CH}_{2} \mathrm{CH}_{2} \mathrm{CH}_{2} \mathrm{OH}\right.$, $74.12 \mathrm{~g} / \mathrm{mol}$ ) in order to enrol a subject sample as homogeneous as possible. N-butanol, in fact, is considered a neutral smell and is commonly used for the selection of olfactory panel members. Each subject was asked to identify the N-butanol solution, prepared at different concentrations, from the distilled water. The lowest concentration 
was tested first, then the concentration was increased until the subject was able to distinguish the odorant from the water. The concentration level at which the N-butanol was identified corresponded to the olfactory perception threshold of the subject. We recruited only subjects who exhibited a similar olfactory perception threshold. The experimental timeline was designed as follows:

- 3 minutes of initial rest;

- 1 minute of pre-stimulus rest;

- 5 seconds of the first olfactory stimulation;

- 1 minute of post-stimulus rest;

- 20 seconds for the self assessment questionnaire (SAM);

- 1 minute of pre-stimulus rest;

- 5 seconds of the second olfactory stimulation;

- 1 minute of post-stimulus rest;

- 20 seconds for the self assessment questionnaire;

- 3 minutes of final rest;

Presentation order of olfactory stimuli was randomized among subjects, and performed between two resting sessions. After each stimulus the participant was asked to score the stimulus in terms of arousal (from 1 to 5) and valence (from -2 to 2) using the Self Assessment Manikin (SAM) test [16].

\subsection{Statistical analysis and Classification Procedure}

Between-group differences were investigated for each feature using non-parametric statistics for paired data (i.e., Wilcoxon signed-rank test). In order to automatically discern whether a subject was experiencing a pleasant or unpleasant olfactory perception using heartbeat dynamics exclusively, we exploited our instantaneous features by feeding an automatic pattern recognition algorithm. This single-subject discrimination of the olfactory pleasantness comprised the following steps: as a pre-processing step, each feature value gathered throughout the olfactory elicitation was normalized by subtracting the corresponding value estimated during the initial resting state; the obtained feature set comprising $\mu_{R R}, \sigma_{R R}^{2}$, LF, HF, LF/HF, HH, LH, LL, $\lambda_{1}, \lambda_{2}, A_{I}$ and $S_{I}$ is taken as an input of a k-Nearest Neighbourhood (KNN)-based classifier. As a validation step, a leave-one-subject-out procedure (LOSO) was applied. Specifically, considering data from $N$ subjects, at each of the $N$ iterations, the training-set comprised data from $(N-1)$ subjects to recognize the valence responses of the $N_{t h}$ subject. This procedure was iterated $N$ times. Within the LOSO scheme, the final, optimal feature-set was obtained identifying the combination of features and classifier parameters (i.e., number of neighbors, $K$ ) that allowed the highest recognition accuracy within the training set. Results of the classification procedure are shown in the form of confusion matrix.

\section{Experimental Results}

Results from the self-assessment-questionnaire showed no significant differences between average arousal scores
$\left(O_{1}: 2.59 \pm 1.34 ; O_{2}: 2.56 \pm 1.22\right.$; Wilcoxon p-value: 0.89). Conversely, significant differences were found along the valence dimension $\left(\mathrm{p}<10^{-6}\right)$, having $O_{1}$ as a pleasant smell (average valence: $0.87 \pm 1.16$ ) and $O_{2}$ as an unpleasant smell $(-1.44 \pm 0.62)$. Therefore, as hypothesized, the chosen odors elicited the same emotional intensity but different pleasantness.

The inhomogeneous point-process nonlinear model was applied to each RR interval series, gathered from each subject. In 24 out of 32 recordings, KS plots and autocorrelation samples fell within $95 \%$ confidence intervals, whereas in the remaining $8 \mathrm{KS}$ plots were slightly outside the boundaries. KS distance analysis reveals a very satisfactory goodness-of-fit, being as low as $0.0284 \pm 0.0064$. According to $\mathrm{KS}$ analysis results, we selected a linear order of 7, quadratic order of 2, and cubic order of 1 . Results from univariate statistical analysis for each instantaneous feature are summarized in Table 1.

Table 1. Median \pm Median absolute deviation of each feature estimated within $5 \mathrm{~s}$ stimuli.

\begin{tabular}{|c|c|c|c|}
\hline Feature & $O_{1}$ & $O_{2}$ & p-val \\
\hline$\mu_{R R}[\mathrm{msec}]$ & $811.64 \pm 121.41$ & $784.41 \pm 96.61$ & 0.94 \\
\hline$\sigma_{R R}^{2}\left[\mathrm{msec}^{2}\right]$ & $682.25 \pm 453.80$ & $439.03 \pm 276.13$ & 0.045 \\
\hline $\mathrm{HF}\left[\mathrm{msec}^{2}\right]$ & $563.15 \pm 456.89$ & $488.64 \pm 361.62$ & 0.28 \\
\hline $\mathrm{LF}\left[\mathrm{msec}^{2}\right]$ & $932.03 \pm 580.25$ & $648.20 \pm 279.20$ & 0.085 \\
\hline $\mathrm{LF} / \mathrm{HF}$ & $1.55 \pm 1.01$ & $1.46 \pm 1.16$ & 0.17 \\
\hline $\mathrm{HH}\left[\mathrm{msec}^{4}\right]$ & $(6.37 \pm 5.63) \mathrm{E}+08$ & $(8.77 \pm 6.76) \mathrm{E}+08$ & 0.28 \\
\hline $\mathrm{LH}\left[\mathrm{msec}^{4}\right]$ & $(4.44 \pm 2.98) \mathrm{E}+08$ & $(2.58 \pm 1.58) \mathrm{E}+08$ & 0.55 \\
\hline $\mathrm{LL}\left[\mathrm{msec}^{4}\right]$ & $(4.28 \pm 3.25) \mathrm{E}+08$ & $(2.94 \pm 2.13) \mathrm{E}+08$ & 0.49 \\
\hline$\lambda_{1}$ & $-0.273 \pm 0.067$ & $-0.270 \pm 0.106$ & 0.93 \\
\hline$\lambda_{2}$ & $-0.356 \pm 0.056$ & $-0.360 \pm 0.071$ & 0.85 \\
\hline$A_{I}$ & $0.348 \pm 0.049$ & $0.314 \pm 0.060$ & 0.076 \\
\hline \multicolumn{2}{|c|}{$0.295 \pm 0.072$} & $0.280 \pm 0.063$ & 0.12 \\
\hline \multicolumn{2}{|c|}{ p-values refer to the Wilcoxon signed-rank test between $O_{1}$ and $O_{2}$}
\end{tabular}

Exemplary estimation of the instantaneous dominant Lyapunov exponent across all subjects is shown in Figure 1. Results from the best odor pleasantness classification are shown in Table 2. This included information from the instantaneous LF power, instantaneous Lyapunov ex-

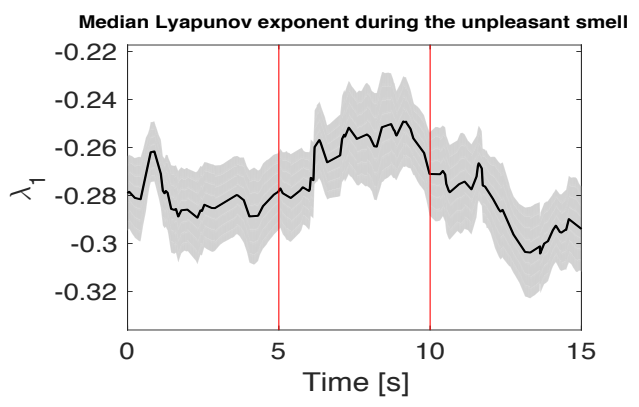

Figure 1. First Lyapunov exponent dynamics before, during and after the stimulus $\mathrm{O} 2$. 
ponents $\left(\lambda_{1}, \lambda_{2}\right)$, and instantaneous approximate entropy, as revealed by the feature selection procedure. Our instantaneous heartbeat linear and nonlinear features estimated within the $5 \mathrm{~s}$ window elicitation were able to discern the two odors with a balanced accuracy of $71.88 \%$, with specificity and a sensitivity of $68.75 \%$ and $75 \%$ respectively.

Table 2. Confusion Matrix of olfactory pleasantness classification using instantaneous LF power, Lyapunov exponents $\left(\lambda_{1}, \lambda_{2}\right)$, and approximate entropy.

\begin{tabular}{c|cc} 
& Positive & Negative \\
\hline Positive & $68.75 \%$ & $25 \%$ \\
Negative & $31.25 \%$ & $75 \%$
\end{tabular}

\section{Discussion and Conclusions}

We proposed a methodology to automatically recognize the pleasantness of an affective olfactory stimulus using ultra-short series of heartbeat dynamics exclusively. We applied our recently proposed inhomogeneous pointprocess nonlinear framework to derive instantaneous correlates of linear and nonlinear ANS activity on cardiovascular control in data gathered from 32 healthy subjects (16 males) undergoing pleasant and unpleasant olfactory elicitation. Of note, each olfactory elicitation lasted only $5 \mathrm{~s}$. To this end, instantaneous measures defined in the time and frequency domains, as well as instantaneous estimates of heartbeat nonlinear/complex dynamics were derived.

Subjective ratings on SAM questionnaires confirmed that the benzaldehyde was perceived as pleasant, whereas the lsovaleric acid was perceived as unpleasant. No significant differences were found along the arousal dimension. Group-wise statistics revealed that higher heartbeat dynamics variance (which may be linked to parasympathetic activity) seems to be associated with pleasant odor, whereas unpleasant odors tend to decrease instantaneous heartbeat complexity (uncorrected p-values). At a singlesubject level, a feature selection procedure highlighted that major discriminant information lies in heartbeat complexity. Using a KNN algorithm, a satisfactory recognition accuracy of $71.88 \%$ was achieved in discerning the pleasant/unpleasant odor perception.

In conclusion, we demonstrated that it is possible to address the challenging task of discerning valence levels of olfactory elicitation using cardiovascular information exclusively, particularly linked to time-varying heartbeat complexity. To our knowledge, the proposed processing approach is unique in quantifying time-varying nonlinear/complex estimates heartbeat dynamics, therefore allowing estimation within an ultra short-time window (i.e., $5 \mathrm{sec}$, coinciding with the exposure to the olfactory stimulus) and to overcome the methodological limitations of previous studies. Future endeavours will focus on the investigation of optimal classification methods, as well as further odorants and cardiovascular estimates. Moreover, the relationship between cardiovascular and other physi- ological dynamics, including electrodermal activity, respiration, EEG, etc., during hedonic olfactory elicitations will be investigated as well.

\section{Acknowledgements}

This research has received partial funding from European Commission - Horizon 2020 Programme grant $n$ 689691 "NEVERMIND".

\section{References}

[1] Gulas CS, Bloch PH. Right under our noses: Ambient scent and consumer responses. Journal of Business and Psychology 1995;10(1):87-98.

[2] Van Toller S. Emotion and the brain. In Perfumery. Springer, 1988; 121-143.

[3] He W, et al. Implicit and explicit measurements of affective responses to food odors. Chem senses 2016;41(8):661-668.

[4] Igarashi M, Song C, Ikei H, Ohira T, Miyazaki Y. Effect of olfactory stimulation by fresh rose flowers on autonomic nervous activity. The Journal of Alternative and Complementary Medicine 2014;20(9):727-731.

[5] Bensafi M, et al. Autonomic nervous system responses to odours: the role of pleasantness and arousal. Chemical Senses 2002;27(8):703-709.

[6] Robin O, et al. Basic emotions evoked by eugenol odor differ according to the dental experience. a neurovegetative analysis. Chemical senses 1999;24(3):327-335.

[7] Vernet-Maury E, et al. Basic emotions induced by odorants: a new approach based on autonomic pattern results. Journal of the autonomic nervous system 1999;75(2):176-183.

[8] Herz RS. Aromatherapy facts and fictions: a scientific analysis of olfactory effects on mood, physiology and behavior. Int J of Neuroscience 2009;119(2):263-290.

[9] Alaoui-Ismaili O, et al. Odor hedonics: connection with emotional response estimated by autonomic parameters. Chemical senses 1997;22(3):237-248.

[10] Kroupi E, et al. Subject-independent odor pleasantness classification using brain and peripheral signals. IEEE Transactions on Affective Computing 2016;7(4):422-434.

[11] Valenza G, et al. Point-process nonlinear models with laguerre and volterra expansions: Instantaneous assessment of heartbeat dynamics. Signal Processing IEEE Transactions On 2013;61(11):2914-2926.

[12] Valenza G, et al. Inhomogeneous point-process entropy: An instantaneous measure of complexity in discrete systems. Physical Review E 2014;89(5):052803.

[13] Valenza G, et al. Estimation of instantaneous complex dynamics through lyapunov exponents: a study on heartbeat dynamics. PloS one 2014;9(8):e105622.

[14] Citi L, et al. A real-time automated point-process method for the detection and correction of erroneous and ectopic heartbeats. Biomedical Engineering IEEE Transactions on 2012;59(10):2828-2837.

[15] Naudin M, et al. State and trait olfactory markers of major depression. PLoS One 2012;7(10):e46938.

[16] Bradley MM, Lang PJ. Measuring emotion: the selfassessment manikin and the semantic differential. Journal of behavior therapy and experimental psychiatry 1994; 25(1):49-59. 